\title{
Velocity of sound in a Bose-Einstein condensate in the presence of an optical lattice and transverse confinement
}

\author{
M. Krämer ${ }^{1,2}$, C. Menotti ${ }^{1,2}$, and M. Modugno ${ }^{2,3}$ \\ ${ }^{1}$ Dipartimento di Fisica, Università di Trento, I-38050 Povo, Italy \\ ${ }^{2}$ BEC-INFM Trento, I-38050 Povo, Italy \\ ${ }^{3}$ LENS - Dipartimento di Fisica, Università di Firenze and INFM, \\ I-50019 Sesto Fiorentino, Italy
}

\begin{abstract}
We study the effect of the transverse degrees of freedom on the velocity of sound in a Bose-Einstein condensate immersed in a one-dimensional optical lattice and radially confined by a harmonic trap. We compare the results of full three-dimensional calculations with those of an effective $1 D$ model based on the equation of state of the condensate. The perfect agreement between the two approaches is demonstrated for several optical lattice depths and throughout the full crossover from the 1D mean-field to the Thomas Fermi regime in the radial direction.
\end{abstract}

Ever since the achievement of Bose-Einstein condensation (BEC) with atomic vapors, an extensive activity in the study of the excitations of these systems has been carried out. A particular example is the exploration of sound excitations in elongated condensates 12345. The propagation of sound in one-dimensional (1D) optical lattices has been the subject of recent studies performed both in the linear 67891011 and nonlinear regimes 12. To capture the main physics, the system can be conveniently described by means of effective 1D theories, which account for the transverse degrees of freedom through a renormalization of the coupling constant. Still, it is important to check the effect of the transverse degrees of freedom since they are known to play a relevant role in some cases 13 .

In this paper, we show that the sound velocity in presence of a 1D lattice and transverse harmonic confinement can be obtained by employing an effective 1D approach, and demonstrate that the effect of the transverse 


\section{Krämer, C. Menotti, and M. Modugno}

harmonic confinement is completely accounted for by the expression $\mathbf{7 8 9 1 1 0}$

$$
c=\sqrt{\frac{N}{m^{*}} \frac{\partial \mu(N)}{\partial N}}
$$

where $N$ is the number of atoms per lattice well and $m^{*}$ is the effective mass. The effect of lattice and transverse confinement both on the equation of state $\mu(N)$ and $m^{*}$ is discussed in detail.

Let us consider an axially symmetric condensate, which is radially confined by the potential $V_{\mathrm{ho}}=m \omega_{\perp}^{2} r^{2} / 2$, and immersed in a $1 \mathrm{D}$ periodic potential of the form $V_{\text {lat }}(z)=s E_{R} \sin ^{2}(\pi z / d)$, where $d$ is the lattice period, $E_{R}=q_{B}^{2} / 2 m$ the recoil energy, $q_{B}=\hbar \pi / d$ the Bragg-momentum, and $s$ the lattice depth in units of the recoil energy. We consider any additional axial confinement to be negligible, so that the Gross-Pitaevskii (GP) equation takes the form

$$
\left[-\frac{\hbar^{2}}{2 m} \nabla^{2}+V_{\text {ho }}(r)+V_{\text {lat }}(z)+g N|\Psi(r, z)|^{2}\right] \Psi(r, z)=\mu \Psi(r, z),
$$

where $N$ is the number of atoms per lattice well and the condensate wavefunction is normalized according to $\int d^{2} r \int_{-d / 2}^{d / 2} d z|\Psi(r, z)|^{2}=1$.

In the linear regime, the small oscillations around the condensate ground state can be described by expanding the order parameter as $\Phi(r, z, t)=$ $e^{-i \mu t / \hbar}\left[\Psi(r, z)+\sum_{j \nu q} u_{j \nu q} e^{-i \omega_{j \nu}(q) t}+v_{j \nu q}^{*} e^{i \omega_{j \nu}(q) t}\right]$, where the functions $u_{j \nu q}$ and $v_{j \nu q}$ are the Bogoliubov quasiparticle amplitudes of quasimomentum $q$, and quantum numbers $j, \nu$ representing the band index and the number of radial nodes respectively. In particular, the lowest energy solutions, with $j=1$ and $\nu=0$, are axial phonons that propagate with the sound velocity $c$. The latter is defined as the slope of the lowest branch in the excitation spectrum $\omega_{j \nu}(q)$, through the relation $c=\hbar \partial \omega_{10}(q) /\left.\partial q\right|_{q=0}$. Therefore it can be obtained by numerically solving the spectrum of the full $3 \mathrm{D}$ system, as discussed in 13 .

In the following we will show that the system can be equivalently described by an effective 1D approach. The basic assumption we make is that the condensate wavefunction can be factorised into a longitudinal and a radial part $\Psi(r, z)=\varphi(r) \psi(z)$. The physics underlying this assumption is that interactions mainly affect the radial wavefunction $\varphi(r)$, while the axial wavefunction $\psi(z)$ is that of a single particle in the periodic potential. This factorization is exact for $s=0$, where the system is uniform in the longitudinal direction. In the case $s \neq 0$, the solution of the GP equation for a transversally uniform system shows that for the densities of many current experiments 14151617 the equation of state and the effective mass can be calculated neglecting the density dependence of $\psi(z)$ 9. The remaining relevant 


\section{Velocity of sound in a Bose-Einstein condensate in an optical lattice}

density dependence of these quantities arises from their explicit dependence on the number of particles (see below). The range of densities for which the factorization ansatz is correct becomes larger as the lattice depth $s$ is increased.

Under the factorization assumption, and using the normalisation condition for the wavefunction, one can write two effective GP equations

$$
\begin{aligned}
& {\left[-\frac{\hbar^{2}}{2 m} \nabla_{r}^{2}+V_{\mathrm{ho}}(r)+\left(g \int_{-d / 2}^{d / 2}|\psi(z)|^{4} d z\right) N|\varphi(r)|^{2}\right] \varphi(r)=\mu_{\perp} \varphi(r),} \\
& {\left[-\frac{\hbar^{2}}{2 m} \nabla_{z}^{2}+V_{\text {lat }}(z)+\left(g \int|\varphi(r)|^{4} d^{2} r\right) N|\psi(z)|^{2}\right] \psi(z)=\mu_{z} \psi(z),}
\end{aligned}
$$

where $N$ is the number of particles in each well at equilibrium, $\mu_{z}=\mu-\epsilon_{\text {ho }}$ and $\mu_{\perp}=\mu-\epsilon_{\text {lat }}$, being $\epsilon_{\mathrm{ho}}=\int d^{2} r \varphi^{*}(r)\left[-\frac{\hbar^{2}}{2 m} \nabla_{r}^{2}+V_{\mathrm{ho}}(r)\right] \varphi(r)$ and $\epsilon_{\text {lat }}$ the single particle ground state energy of the periodic potential.

The effective mass is calculated from the lowest energy Bloch band

$$
\varepsilon(k)=\int_{-d / 2}^{d / 2} \psi_{k}^{*}(z)\left[-\frac{\hbar^{2}}{2 m} \nabla_{z}^{2}+V_{\mathrm{lat}}(z)+\frac{g \tilde{N}}{2 a_{\perp}^{2}}\left|\psi_{k}(z)\right|^{2}\right] \psi_{k}(z) d z,
$$

according to the general relation $1 / m^{*}=\partial^{2} \varepsilon /\left.\partial k^{2}\right|_{k=0}$. 9. In Eq. (5.5) the explicit dependence of the energy per particle on density appears through the renormalized number of atoms $\tilde{N}=N a_{\perp}^{2} \int|\varphi(r)|^{4} d^{2} r$, where $a_{\perp}=$ $\sqrt{\hbar / m \omega_{\perp}}$. Consistently with the above discussion, the Bloch state $\psi_{k}(z)$ at quasi-momentum $k$ is taken as the single particle solution of (4). Then $\psi_{k}(z)$ is independent of the radial confinement and the effect of the radial trapping on $m^{*}$ is captured by its dependence on the renormalized number of atoms $\tilde{N}$.

Equation (3) describes a BEC uniform along $z$ and harmonically confined along $r$ (infinite cylinder). The effect of the lattice is included in the effective coupling constant

$$
\tilde{g}=g d \int_{-d / 2}^{d / 2}|\psi(z)|^{4} d z,
$$

where again $\psi(z)$ is the single particle ground state of (4). Without radial confinement, in a system with $3 \mathrm{D}$ average density $n$, the equation of state $\mu_{\perp}(n)=\tilde{g} n$ is linear in the density, since $\tilde{g}$ depends strongly on the optical lattice depth, but not on density. As mentioned above, this result is known to be correct in the presence of a lattice for sufficiently small densities 9 .

In order to discuss the effect of the transverse degrees of freedom, we concentrate now on the radial dynamics. The presence of the lattice is described by the effective coupling constant $\tilde{g}$, so that the crossover from frozen 


\section{Krämer, C. Menotti, and M. Modugno}

radial dynamics (1D mean-field) to the Thomas-Fermi (TF) regime, can be described in full analogy to the case of the infinite cylinder 18. Introducing the dimensionless variable $\rho=r / a_{\perp}$ and defining $f(\rho)=a_{\perp} \varphi(r)$, Eq. (33) can be rewritten in the dimensionless form

$$
\left[-\frac{1}{2} \nabla_{\rho}^{2}+\frac{1}{2} \rho^{2}+4 \pi \tilde{a} n_{1 D}|f(\rho)|^{2}\right] f(\rho)=\frac{\mu_{\perp}}{\hbar \omega_{\perp}} f(\rho)
$$

where $n_{1 D}=N / d$ is the linear average density, $\tilde{a}$ is the effective scattering length obtained from the relation $\tilde{g}=4 \pi \hbar^{2} \tilde{a} / m$, and $f$ is normalized according to $\int d^{2} \rho|f|^{2}=1$. Notice that the solution of the above equation depends only on the parameter $\tilde{a} n_{1 D}$, yielding an exact scaling behavior for the radial wavefunction as a function of the transverse trapping.

The dependence of the chemical potential $\mu_{\perp} / \hbar \omega_{\perp}$ on $\tilde{a} n_{1 D}$ determines the equation of state in the presence of the radial trap. For $\tilde{a} n_{1 D} \gg 1$, the system is in the TF regime and one finds $\mu_{\perp} / \hbar \omega_{\perp}=2\left(\tilde{a} n_{1 D}\right)^{1 / 2}$. In the opposite limit $\tilde{a} n_{1 D} \ll 1$, instead, the radial profile is gaussian and the equation of state is given by $\mu_{\perp} / \hbar \omega_{\perp}=1+2 \tilde{a} n_{1 D}$. Given the solution $\mu_{\perp} / \hbar \omega_{\perp}$ as a function of the universal parameter $\tilde{a} n_{1 D}$, the velocity of sound can be calculated according to Eq.(11). This procedure yields the result $c=\sqrt{g n(0) / 2 m^{*}}$ both in the TF regime $\left(\tilde{a} n_{1 D} \gg 1\right)$ and in the $1 \mathrm{D}$ meanfield limit $\left(\tilde{a} n_{1 D} \ll 1\right)$ 10 . To find these limiting results, we have used the fact that $n_{1 D}=\left(4 \pi^{2} \hbar^{2} \tilde{a} / m^{2} \omega_{\perp}^{2}\right) n^{2}(0)$ in the TF regime and $n_{1 D}=\pi a_{\perp}^{2} n(0)$ in the $1 \mathrm{D}$ mean-field limit.

The result for the sound velocity in the intermediate regimes can be calculated numerically, just based on the knowledge of the following quantities: (i) the renormalized scattering length $\tilde{a}$ for any given value of $s$, obtained from a $1 \mathrm{D}$ calculation of the single particle ground state solution in presence of the lattice; (ii) the equation of state and $\tilde{N}$ for the infinite cylinder at any $\tilde{a} n_{1 D}$; (iii) the effective mass $m^{*}$ at any given value of $s$ for the renormalised number of atoms per well $\tilde{N}$, obtained from Eq. (15) by a $1 \mathrm{D}$ calculation of the single particle Bloch solutions. This procedure leads to the results shown in Fig [1, where the theoretical predictions are compared with the results of the full 3D numerical calculations for several values of the lattice depth. The agreement is perfect in the full crossover.

Furthermore, it is interesting to note that by relating $n_{1 d}$ to the density at the center $n(0)$ one can show that the value $c=\sqrt{\tilde{g} n(0) / 2 m^{*}}$ provides an estimate for the sound velocity which is correct within $5 \%$ throughout the whole crossover from TF to 1D.

We also notice that in the TF regime, $\tilde{a} n_{1 D} \gg 1$, the sound velocity in presence of transverse harmonic confinement corresponds to that of a transversally uniform condensate with the average density $\bar{n}, c=\sqrt{\tilde{g} \bar{n} / 2 m^{*}}$. 


\section{Velocity of sound in a Bose-Einstein condensate in an optical lattice}

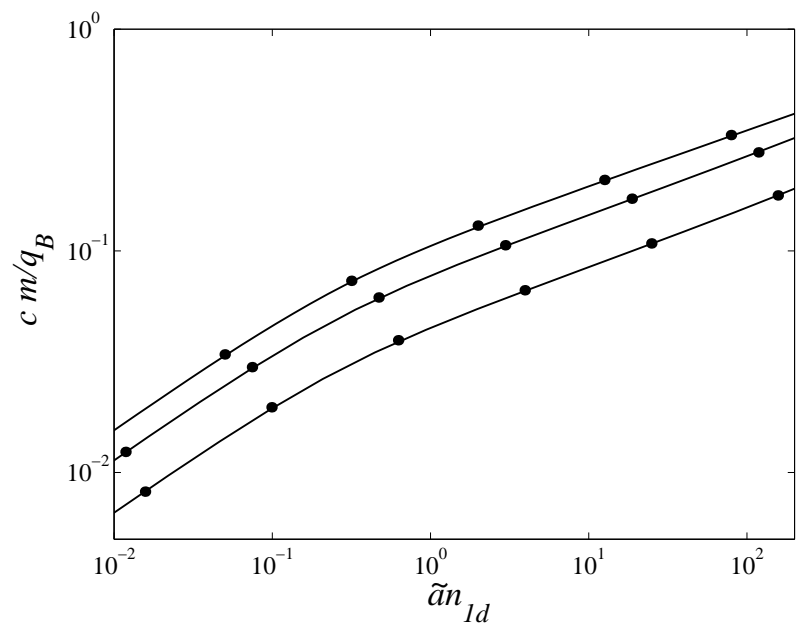

Fig. 1. Velocity of sound as a function of $\tilde{a} n_{1 D}$, for three values of the lattice depth (from top to bottom $s=0,5,10$ ). Results of full 3D numerical calculations (points) are compared with the prediction derived from the 1D equation of state (lines). Note that $\tilde{a} / a=1.49$ and $\tilde{a} / a=1.98$ for $s=5$ and $s=10$ respectively.

In fact, in this regime the radial profile is an inverted parabola, leading to an average density $\bar{n} \equiv \int d x d y n(x, y) / \pi d R^{2}$ equal to half of the central density $n(0)$ 345. This explanation in terms of $\bar{n}$ works well in the TF regime, and to some extent also in the 1D mean-field regime, where the definition for the average density is replaced by $\bar{n} \equiv \int d x d y n^{2}(x, y) / \int d x d y n(x, y)$ 19. However, since the concept of average density is not well defined in general, it does not provide a general criterion for the determination of the sound velocity in the intermediate regimes.

In conclusion, we have demonstrated that the equation of state of an infinite cylinder captures the effect of the transverse degrees of freedom on the sound velocity in a condensate immersed in a $1 \mathrm{D}$ optical lattice, upon a suitable renormalization of the mass and of the coupling constant. This result is obtained by means of a factorization ansatz for the condensate wavefunction. The predictions of the factorized model yield perfect agreement with full 3D numerical calculations in a wide range of optical lattice depths and in the full crossover from the $1 \mathrm{D}$ mean field regime to the $\mathrm{TF}$ limit. The factorization ansatz rely on the assumption that in absence of radial trapping the equation of state is linear in the number of particles. This condition is always fulfilled at $s=0$ and in the tight binding regime, while for intermediate $s$ it is satisfied to a good approximation at experimentally relevant densities. In any case, Eq.(11) is completely general, and could be 


\section{Krämer, C. Menotti, and M. Modugno}

easily checked also beyond the limit of validity of the factorization ansatz. In that case the determination of $\mu(N)$ would require from the beginning a full 3D calculation. Instead, when the factorization assumption holds, the simple model presented in this paper can be used to extract the correct value of the sound velocity.

\section{ACKNOWLEDGMENTS}

This research is partially supported by the Ministero dell'Istruzione, dell'Università e della Ricerca (MIUR). We thank Franco Dalfovo, Lev Pitaevskii, Augusto Smerzi and Sandro Stringari for useful discussions.

\section{REFERENCES}

1. M.R. Andrews, D.M. Kurn, H.-J. Miesner, D.S. Durfee, C.G. Townsend, S. Inouye, and W. Ketterle, Phys. Rev. Lett. 79, 553 (1997).

2. M.R. Andrews, D.M. Stamper-Kurn, H.-J. Miesner, D.S. Durfee, C.G. Townsend, S. Inouye, and W. Ketterle, Phys. Rev. Lett. 80, 2967 (1998).

3. E. Zaremba, Phys. Rev. A 57, 518 (1998).

4. G.M. Kavoulakis and C.J. Pethick, Phys. Rev. A, 581563 (1998).

5. S. Stringari, Phys. Rev. A 58, 2385 (1998).

6. M. Krämer, L. Pitaevskii and S. Stringari, Phys. Rev. Lett. 88, 180404 (2002).

7. M. Machholm, C.J. Pethick, and H. Smith, Phys. Rev. A 67, 053613 (2003).

8. A. Smerzi and A. Trombettoni, Phys. Rev. A 68, 023613 (2003).

9. M. Krämer, C. Menotti, L. Pitaevskii and S. Stringari, Eur. Phys. J. D 27, 247 (2003).

10. E. Taylor and E. Zaremba, Phys. Rev. A 68, 053611 (2003).

11. J.-P. Martikainen and H.T.C. Stoof, Phys. Rev. A 69, 023608 (2004).

12. C. Menotti, M. Krämer, A. Smerzi, L. Pitaevskii, and S. Stringari, Phys. Rev. A 70, 023609 (2004).

13. M. Modugno, C. Tozzo, and F. Dalfovo, cond-mat/0405653 Phys. Rev. A, in press.

14. J.H. Denschlag, J.E. Simsarian, H. Häffner, C. McKenzie, A. Browaeys, D. Cho, K. Helmerson, S.L. Rolston and W.D. Phillips, J. Phys. B 35, 3095 (2002).

15. F.S. Cataliotti, S. Burger, C. Fort, P. Maddaloni, F. Minardi, A. Trombettoni, A. Smerzi and M. Inguscio, Science 293, 843 (2001).

16. O. Morsch, M. Cristiani, J.H. Müller, D. Ciampini, and E. Arimondo, Phys. Rev. A 66, 021601 (2002).

17. M. Greiner, I. Bloch, O. Mandel, T.W. Hänsch, and T. Esslinger, Phys. Rev. Lett. 87, 160405 (2001).

18. C. Menotti and S. Stringari, Phys. Rev. A 66, 043610 (2002).

19. A.D. Jackson, G.M. Kavoulakis and C.J. Pethick, Phys. Rev.A 58, 2417 (1998). 